\title{
KDD for BSN - Towards the Future of Pervasive Sensing
}

\author{
Guang-Zhong Yang \\ Imperial College London
}

\begin{abstract}
With increasing sophistication and miniaturisation of wireless sensor technologies, integrated microsensors no more than a few millimetres in size combined with onboard processing and wireless data transfer has become a reality. The provision of ubiquitous and pervasive monitoring of physical, physiological, and biochemical parameters in any environment and without activity restriction and behaviour modification is the primary motivation of Body Sensor Network (BSN) research. The general scope of BSN is broad, ranging from monitoring of patients with chronic disease and care for the elderly, to general well-being monitoring and performance evaluation in sports. It also has important applications in gaming and human-computer-interaction. One of the significant challenges of BSN is the provision of context aware sensing with effective multi-sensor fusion, data inferencing, mining, and trend analysis. Other research issues currently being addressed include novel miniaturised bioelectrical, biochemical, biophysical, and mechanical sensors; low power RF transceiver, energy scavenging, and battery technologies; biocompatibility, materials, system integration and miniaturisation; autonomic sensor networks and light-weight communication protocols and standards. This talk will address some of the key research topics and current advances in BSN, particularly those related to the KDD community. It will also cover the use of bio-inspired design for providing distributed inferencing and ultra-low power on-node processing, demonstrating how this alternate paradigm based on the strategies used by biological systems can be used to deal with the challenges of scale, complexity, heterogeneity, and uncertainty involved in pervasive sensing.
\end{abstract}

